\title{
State Dependent Multicast Routing for Single Rate Loss Networks *
}

\author{
Chi-Chung Cheung, Danny H.K. Tsang \\ Department of Electrical and Electronic Engineering \\ Hong Kong University of Science and Technology \\ Clear Water Bay, Kowloon, Hong Kong \\ Phone: (852) 23587080 _ Fax: (852) 23581485 \\ email: (eeccc, eetsang)@ee.ust.hk \\ Sanjay Gupta \\ Department of Electrical and Computer Engineering \\ Illinois Institute of Technology \\ Chicago, IL 60616, U.S.A. \\ email: gupta@ece.iit.edu
}

\begin{abstract}
In this paper, we investigate a state dependent multicast routing algorithm called Least Load Multicast Routing (LLMR), for single rate loss networks. The algorithm is based on Least Load Routing (LLR) concept and the approach is to select the least load links for establishing connections. To facilitate tractable analysis, the networks considered are assumed symmetrical and fully connected. In addition, connection requests are Poisson arrival and the holding times of accepted calls are exponentially distributed. We combine one-time simulations and fixed point equation (FPE) to develop an analytical model for LLMR. Analytical results are compared with simulation results and the agreement is surprisingly good. We find that the effect of link independence assumption is insignificant for the analytical model.
\end{abstract}

\section{Introduction}

Multicasting refers to the ability of a set of more than two nodes or end-users in a com-

*This research was supported in part by Hongkong Telecom Institute of Information Technology grant: HKTIIT 93/94.EG01. munication network to communicate simultaneously with each other. Applications that require multicast capability (either point-to-multipoint (PTM) as in distributional video or multipointto-multipoint (MTM) as in video conferencing, online collaboration and others) will be an integral part of future broadband services. Given the popularity of multicast end-user services and applications, we study the problem of multicast call routing in single rate loss networks, in which a connection requires a unit capacity on each link that it is routed. If a route cannot be assigned to a connection request, it is assumed that the request is lost and does not retry. Examples of single rate loss networks include telephone networks and homogeneous VP-based ATM networks.

Recent research efforts in the area of multicast call routing have followed the following heuristic approach: the underlying network is modeled as a graph (directed or undirected) with links as edges and switches as nodes; and each edge is assigned a "cost" (for instance the cost could be a measure of expected delay). The multicast routing problem is then reduced to finding a "tree" that spans the nodes that wish to participate in a given multicast connection that has minimal cost [9] [6] [1] [13] [14]. The above problem is known in the literature as the Steiner tree prob- 
lem (an NP-complete problem [10]) for which several heuristics have been proposed. The above routing schemes cannot exploit the inherent statistical nature of network traffic to achieve higher network utilization or reduce call blocking probability. Additionally, given that the above studies assume that routing is done on a packet by packet basis limits their applicability to networks where routing is done on a per circuit or virtual circuit basis.

Given the limitations of the above studies, we consider the problem of developing routing policies for multicast connections that do not decide routes to use beforehand, but take into account the state of the network at the instant a connection request arrives to assign a route. In this study, we consider a circuit switched or a virtual circuit based packet switched network where all connection requests have the same bandwidth requirement. The arrival process of connection requests is assumed to be a random process. Each connection request requires the set up of a multicast connection among a set of randomly chosen nodes. Each connection request, if accepted, departs from the network after a random amount of call holding time. Some initial work in the area of state dependent multicast routing schemes in (single rate) circuit switched networks have been reported in [7] [5].

In this paper, we investigate a state dependent multicast routing algorithm called Least Load Multicast Routing (LLMR), to establish connections by considering the link load of links in single rate loss networks. State dependent ${ }^{1}$ routing of calls (point-to-point) has long been regarded in the telephone industry as a means of increasing call throughput and robustness in the telephone network. We would like to refer the reader to [3] [8] [2] [11] [12] [4] for some of the popular state dependent routing algorithms. A key part of the paper pertains to the development of an analytical model for performance evaluation of the routing algorithm. For this purpose, we also use the well known and often used "link independence assumption" to develop a set of fixed point equations from which a number of performance metrics of interest pertaining to the routing algorithm can be obtained. The analytical results developed are used to engineer routing policies to

\footnotetext{
${ }^{1}$ State refers to a set of variables that describe completely the current status of the network.
}

achieve design objectives (for instance maximize revenue, utilization, and/or meet fairness criteria) and dimension networks.

The paper is organized as follows. We begin by presenting in Section 2 the system model and the state dependent multicast routing algorithm. In Section 3 we develop an analytical model to estimate the performance of the routing algorithm. Section 4 presents the numerical results of the model and show its accuracy. Finally, we conclude in Section 5 by summarizing the paper and outlining avenues for future research.

\section{State Dependent Multi- cast Routing Algorithm}

In LLMR, the information used to make a routing decision is based on the states of links, i.e the number of free circuits of links. To establish a connection, the LLMR algorithm tries to (i) use direct links to route the connection request and (ii) maximize network capacity for future connection requests. In order to achieve the above objectives, the LLMR locates a spanning tree consisting of only direct links with the highest possible states. If no spanning tree is available, the connection request is denied.

To illustrate the routing algorithm outlined above, we consider a 4-node network and a connection request with destination set $\{r, a, b, c\}$ with node $r$ as the source node. The steps in the connection establishment procedure are as shown in Figure 1 - the number next to a link denotes its state and links that are included in the connection request are drawn with "thick" lines. Node $c$ is the first one to be added as link $(r, c)$ has 3 free circuits that is larger than that of links $(r, a)$ and $(r, b)$ which is followed by node $a$ (link $(c, a)$ is chosen due to its larger number of free circuits over links $(r, a),(r, b)$ and $(c, b))$. Finally, we choose link $(a, b)$ because the number of free circuits of $(a, b)$ is the largest compared with links $(r, b)$ and $(b, c)$. After iteration 4 , the procedure is completed and a tree is established for the connection request. 


\section{$3 \quad$ Performance Modeling}

In this section, we develop an analytical model for state dependent multicast routing. The analytical model is to combine one-time simulations and fixed point equation (FPE) to solve for the solution of the problem. FPE is an iterative procedure that alternates between the evaluation of link occupancy distributions and state dependent arrival rates, and hopefully a consistent solution is finally converged. Once the consistent set of link occupancy distributions and state dependent arrival rates of links are obtained, the call blocking probability of each type of connection requests can be calculated.

Consider a fully connected symmetrical network with $N$ nodes and each link having a capacity of $C$ units. Requests for connections (assumed for the discussion here to have identical bandwidth requirements of 1 bandwidth unit) with destination set of size $d, d>1$, arrive according to a Poisson process with rate $\lambda_{d}$. It is assumed that the holding times of connections are independent and identically distributed random variables with unit mean. Further, it is also assumed that the probability that a given node belongs to a destination set is the same for all nodes. Since the network we consider is symmetrical in all respects, the occupancy distributions for all links are identical. Henceforth, we refer to a link as being in state $i, i=0, \ldots, C$, if its occupied capacity is $i$. Let $\pi_{i}, i=0, \ldots, C$, be the probability of the link occupancy being $i$ in steady state. Further, let $\Lambda_{i}, i=0, \ldots, C$, denote the arrival rate of connections to a link in state $i$.

\subsection{Evaluation of link occupancy distributions given state de- pendent arrival rate of links}

Given the state dependent arrival rates of a link, the steady link occupancy distribution can be determined by the solution of a state dependent one dimensional birth-death process as:

$$
\pi_{i}=\frac{\prod_{j=0}^{i-1} \Lambda_{j} / i !}{1+\sum_{j=1}^{C} \prod_{k=0}^{j-1} \Lambda_{k} / j !}, \quad i=0, \ldots, C .
$$

Let $\Lambda=\left(\Lambda_{0}, \ldots, \Lambda_{C-1}\right)$. The above equation to calculate $\pi_{i}$ from $\Lambda$ can be written as:

$$
\pi_{i}=f_{i}(\Lambda), \quad i=0, \ldots, C .
$$

\subsection{Evaluation of state dependent arrival rates of links given link occupancy distributions}

Before proceeding the evaluation, we need to introduce some additional concepts and definitions. Denote by $\Phi(i)$ the probability of a link being in state $i$ or higher, i.e.,

$$
\Phi(i)=\sum_{j=i}^{C} \pi_{j}, \quad i=0, \ldots, C .
$$

Let $Q_{i}(l, m, n)$ be the probability that given $n-1$ links, $l$ links are in the set of states $\{0, \ldots, i-1\}, m-1$ are in state $i$, and the remaining are in the set of states $\{i+1, \ldots, C\}$. A routine combinatorial argument yields

$Q_{i}(l, m, n)=K_{l, m, n}[1-\Phi(i)]^{l} \pi_{i}^{m-1} \Phi(i+1)^{n-l-m}$

where $K_{l, m, n}=\frac{(n-1) !}{l !(m-1) !(n-l-m) !}$ and $l+m \leq n$.

We now consider the following process for building a random graph. We start with an empty graph (i.e., with no edges) over $n$ nodes to which edges are added randomly one at a time as described. The edges are chosen randomly (from the set of all possible edges) one at a time and considered for inclusion in the graph. An edge is discarded (and not considered again) if it forms a cycle, otherwise it is added to the graph. The procedure is stopped as soon as a spanning tree is obtained. Let $q(n, k)$ denote the probability that the $k$ th edge chosen is included in the graph. Similarly, we define $r(n, k), k \geq 0$, to be the probability that a graph over $n$ nodes and having $k$ randomly chosen links is not connected. Table 1 and Table 2 provide numerical values for $q(n, k)$ and $r(n, k)$ obtained through simulations for various graph sizes. It is important to observe that both $q(n, k)$ and $r(n, k)$ are functions of only the number of nodes and links and can therefore be computed "off-line" and stored in "look-up" tables.

It is instructive to note that the LLMR algorithm can be considered as one where links are considered in decreasing order of their residual capacity. A link is chosen for inclusion in the routing tree if it does not form a cycle with the previously included links. Now consider a connection request with a destination set of size $d$. Denote by $Z(d)$ the total number of links to be 
considered in a connection request with destination set of size $d$; for fully connected networks, $Z(d)=d(d-1) / 2$. The probability that a link in state $i$ is the $k$ th $, k=1, \ldots, Z(d)$, one to be considered (recall, links can be assumed as being considered in LLMR for inclusion in the routing tree in order of increasing occupancy with any ties being broken randomly) is

$$
\Psi_{i}(k, d)=\sum_{l=0}^{k-1} \sum_{m=k-l}^{Z(d)-l} \frac{1}{m} Q_{i}(l, m, Z(d))
$$

where $k=1, \ldots, Z(d)$ and $d=2, \ldots, D$, and $D$ is the maximum destination size of all connection requests $(D \leq N)$.

The probability that a link in state $i$ is the $k$ th one to be considered and included in the routing tree for a connection request with destination set of size $d$ is

$$
\theta_{i}(k, d)=q(d, k) \Psi_{i}(k, d)-p_{b}(k, d)
$$

where $p_{b}(k, d)=$

$$
\sum_{l=0}^{Z(d)-k} r(d, k+l)\left(\begin{array}{c}
Z(d)-k \\
l
\end{array}\right)\left(1-\pi_{C}\right)^{l} \pi_{C}^{Z(d)-k-l}
$$

$k=1, \ldots, Z(d)$ and $d=2, \ldots, D$.

Further, since given a destination set size all nodes have the same probability for being chosen as a destination, the total traffic offered to a link in state $i$ is

$$
\Lambda_{i}=\sum_{d=2}^{D} \frac{Z(d)}{Z(N)} \lambda_{d} \sum_{k=1}^{Z(d)} \theta_{i}(k, d), \quad D \leq N .
$$

Note that $\frac{Z(d)}{Z(N)}$ is the probability that a link is chosen when a connection request with destination set of size $d$ arrives.

Let $\pi=\left(\pi_{0}, \ldots, \pi_{C}\right)$. Using Equations (3) to (8), we can obtain $\Lambda_{i}$ from $\pi$ and write:

$$
\Lambda_{i}=g_{i}(\pi), \quad i=0, \ldots, C-1 .
$$

Define class $d$ as the class of connection request with destination set of size $d$. The call blocking probability of class $d$ is

$$
\sum_{k=0}^{Z(d)} r(d, k)\left(\begin{array}{c}
Z(d) \\
k
\end{array}\right)\left(1-\pi_{C}\right)^{k} \pi_{C}^{Z(d)-k},
$$

where $\pi_{C}$ is obtained from the Fixed Point Equations (2) and (9).

\section{Numerical Results}

In this section, we present numerical results to illustrate the performance of the analytical model. We consider a fully connected network with 30 nodes and each link has a capacity of 20 units. Three kinds of connection requests are considered and their destination sizes are 2, 3, and 4 (i.e. $D=4$ ). The ratio of the loading among class 2 , class 3 , and class 4 is $2: 1: 1$ and we define the network loading as the ratio of the total offered load to the total network capacity.

For each simulation result, 10 independent runs are carried out and each run was terminated after $10^{5}$ connection requests have been generated. The initial $10 \%$ of each run was discarded to avoid the transient effect. The vertical line about each point represents the $95 \%$ confidence interval. For the analytical result, the iterative procedure will be terminated if the difference between the current and the previous values of all $\pi_{i}$ s are less than $10^{-4}$, i.e. $\left|\pi_{i}^{(n)}-\pi_{i}^{(n-1)}\right|<10^{-4}$ for $i=0, \ldots, C$ where $\pi_{i}^{(n)}$ is the value of $\pi_{i}$ after $n$ iterations. In our numerical example, the iterative procedure always converges to the solution in less than 20 iterations.

Figure 2 shows the accuracy of the analytical model. The agreement between the simulation and the analytical results is surprisingly good. The high accuracy of the call blocking probability for class 2 shows that the calculations of link occupancy distributions and state dependent arrival rates are not much affected by the link independence assumption. We also observe that the accuracy of call blocking probability of a class worsens if its destination size becomes larger. It is because, from Equation (10), the calculation of the call blocking probability of class $d$ is a polynomial of $\pi_{C}$ in degree $Z(d)$. Hence a small error in $\pi_{C}$ will result in a big discrepancy in the call blocking probability.

\section{Conclusions}

In this paper, we have investigated the state dependent multicast routing algorithm for single rate loss networks. An analytical model for the routing algorithm has been developed. We have compared the simulation and analytical results, and found that the agreement is surpris- 
ingly good. We also found that the effect of link independence assumption is insignificant for the calculation of state dependent arrival rates and link occupancy distributions.

Substantial work is already in progress to develop an analytical model of the algorithm with alternative nodes. We are also trying to migrate the algorithm to ATM networks and to develop the corresponding analytical model.

\section{References}

[1] M. H. Ammar, S. Y. Cheung, and C. M. Scoglio. Routing multipoint connections using virtual paths in an ATM network. In Proceedings of the IEEE INFOCOM, pages 98-105, 1993.

[2] G. R. Ash, J. S. Chen, A. E. Frey, and B. D. Huang. Real time network routing in a dynamic class-of-service network. In Proceedings of the International Teletraffic Congress, pages 187-194, 1991.

[3] G. T. Ash, R. H. Cardwell, and R. P. Murray. Design and optimization of networks with dynamic routing. Bell Systems Technical Journal, vol. 60:1787-1820, 1981.

[4] V. P. Chaudhary, K. R. Krishnan, and C. D. Pack. Implementing dynamic routing in local telephone companies of USA. In Proceedings of the International Teletraffic Congress, pages 87-91, 1991.

[5] C.C. Cheung, H.W. Chu, D.H.K. Tsang, and S. Gupta. Least loaded first multicast routing in single rate loss networks. In Proceedings of the IEEE International Conference of Communications, pages 513-517, 1996.

[6] M. Doar and I. Leslie. How bad is naive multicast routing? In Proceedings of the IEEE INFOCOM, pages 82-89, 1993.

[7] R. H. Hwang. Adaptive multicast routing in single rate loss networks. In Proceedings of the IEEE INFOCOM, pages 571-578, 1995.

[8] P. B. Key and G. A. Cope. Distributed dynamic routing schemes. IEEE Communications Magazine, vol. 28:54-64, October 1990.
[9] V. P. Kompella, J. C. Pasquale, and G. C. Polyzos. Multicasting for multimedia communication. In Proceedings of IEEE INFOCOM, pages 2078-2085, 1992.

[10] M. Minoux. A class of combinatorial problems with polynomially solvable large scale set covering/partitioning relaxations. R.A.I.R.O Operations Research, vol. 21(2):105-134, 1988.

[11] D. Mitra, R. J. Gibbens, and B. D. Huang. State-dependent routing on symmetric loss networks with trunk reservations, I. IEEE Transactions on Communications, pages 400-411, February, 1993.

[12] T. J. Ott and K. R. Krishnan. Statedependent routing of telephone schemes and the use of separable routing schemes. In Proceedings of the International Teletraffic Congress, 1985.

[13] H. Tode, Y. Sakai, M. Yamamoto, H. Okada, and Y. Tezuka. Multicast routing algorithm for nodal load balancing. In Proceedings of the IEEE INFOCOM, pages 2086-2095, 1992.

[14] B. M. Waxman. Routing of multipoint connections. IEEE Journal on Selected Areas in Communications, vol. 6:1617-1622, December 1988 .

[15] B. M. Waxman. Performance evaluation of multipoint routing algorithms. In Proceedings of the IEEE INFOCOM, pages 980-986, 1993. 


\begin{tabular}{||c|c|c|c|c||}
\hline \multirow{2}{*}{$k$} & \multicolumn{4}{|c||}{$n$} \\
\cline { 2 - 5 } & 4 & 5 & 6 & 7 \\
\hline 3 & 0.79995 & .091690 & 0.95602 & 0.97367 \\
\hline 4 & 0.20005 & .070236 & 0.84627 & 0.90945 \\
5 & & 0.27763 & 0.66448 & 0.79964 \\
6 & & 0.08411 & 0.32264 & 0.65092 \\
\hline 7 & & 0.01900 & 0.13288 & 0.35876 \\
8 & & & 0.05184 & 0.16901 \\
9 & & & 0.01893 & 0.07668 \\
\hline 10 & & & 0.00582 & 0.03475 \\
11 & & & 0.00112 & 0.01580 \\
12 & & & & 0.00704 \\
\hline 13 & & & & 0.00290 \\
14 & & & & 0.00103 \\
15 & & & & 0.00029 \\
\hline 16 & & & & 0.00005 \\
\hline
\end{tabular}
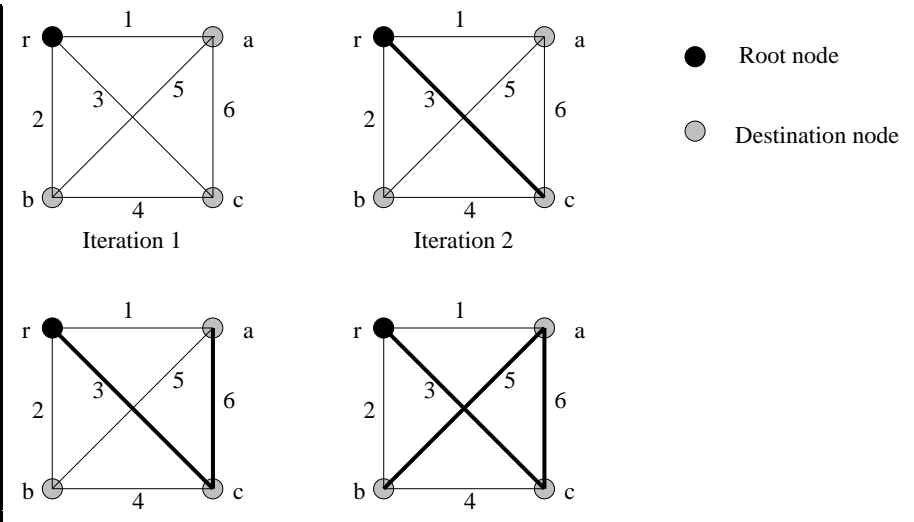

Iteration 3

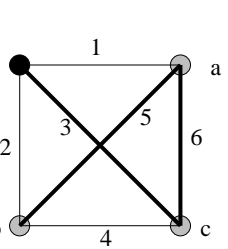

Iteration 4

Figure 1: Example to illustrate LLMR

Table 1: $q(n, k)$ as a function of $n$ and $k$

\begin{tabular}{||c|c|c|c|c||}
\hline \multirow{2}{*}{$k$} & \multicolumn{4}{|c||}{$n$} \\
\cline { 2 - 5 } & 4 & 5 & 6 & 7 \\
\hline 0 & 0.20037 & 0.38098 & 0.50737 & 0.59314 \\
1 & 0.00000 & 0.10310 & 0.20852 & 0.29173 \\
2 & & 0.01895 & 0.07810 & 0.13541 \\
\hline 3 & & 0.00000 & 0.02601 & 0.06108 \\
4 & & & 0.00691 & 0.02689 \\
5 & & & 0.00111 & 0.01142 \\
\hline 6 & & & 0.00000 & 0.00432 \\
7 & & & & 0.00134 \\
8 & & & & 0.00031 \\
\hline 9 & & & & 0.00005 \\
10 & & & & 0.00000 \\
\hline
\end{tabular}

Table 2: $r(n, n-1+k)$ as a function of $n$ and $k$

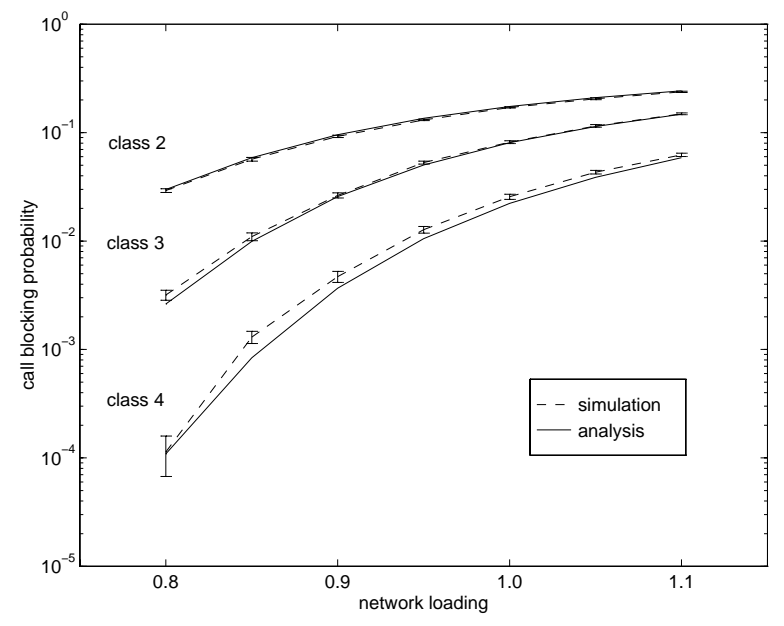

Figure 2: Call blocking probability versus network loading 